\title{
On Stability of Discontinuous Galerkin Time-Domain Method for Conductive Medium
}

\author{
Mehmet Burak Ozakin*, Liang Chen, Shehab Ahmed, and Hakan Bagci \\ Division of Computer, Electrical, and Mathematical Science and Engineering \\ King Abdullah University of Science and Technology (KAUST) \\ Thuwal 23955-6900, Saudi Arabia \\ \{mehmet.ozakin, liang.chen, shehab.ahmed, hakan.bagci\}@kaust.edu.sa
}

In recent years, discontinuous Galerkin time-domain (DGTD) method has found widespread use in computational electromagnetics [1-2]. This is due to the fact that it combines the advantages of finite-element and finite-volume methods (FEM and FVM). Just like FEM, it allows for accurate representation of the geometry and uses higherorder basis functions. Like FVM, it uses numerical flux to realize information exchange between discretization elements localizing all spatial operations. This yields a block diagonal mass matrix, where the dimension of each block is equal to the degrees of freedom in each element. The inverse of mass matrix is computed block by block and stored before the time marching starts. Using an explicit integrator to execute the time marching results in a very efficient and compact DGTD solver. Indeed, high-order (explicit) Runge-Kutta (RK) methods are often incorporated with DG frameworks making use of nodal high-order polynomial basis functions to solve Maxwell equations [2].

In this work, we first study the stability of this type of DGTD methods (termed as RK-DGTD hereinafter) when they are applied to electromagnetic problems involving conductive materials and/or background medium. For the sake of simplicity, we solve the one-dimensional (1D) Maxwell equations in a cavity with perfect electric conductor (PEC) boundaries and loaded with a conductive material. In the RK-DGTD solution, standard stability analysis shows that the eigenvalues of DG system gradually move out of the RK stability region with the increasing conductivity, regardless of the numerical flux type chosen in the DGTD. The upper bound of the timestep size predicted from this analysis matches exactly with the maximum step size that yields a stable solution in the numerical experiments.

To overcome this problem, we propose to incorporate a leap-frog (LF) time integration scheme with the DG method. Inspired from the LF schemes developed for the finite-difference time-domain (FDTD) method [3], we apply time averaging to the electric field term that is multiplied by the conductivity. To study the stability properties of the resulting LF-DGTD scheme, we cast the time updates in the form $F\left(t_{n+1}\right)=G F\left(t_{n}\right)$ [4]. For the solution to be stable, all eigenvalues of matrix $G$ have to be inside the unit circle. Indeed, unlike the RK-DGTD, for LG-DGTD with time averaging, increasing the value of the conductivity does not push these eigenvalues out of the unit circle (stability region). The LF-DGTD is always stable as long as a time step size is chosen based on the CFL condition of the same problem without loss.

1. J. S. Hesthaven and T. Warburton, Nodal Discontinuous Galerkin Methods: Algorithms, Analysis, and Applications, Springer Science \& Business Media, 2008.

2. K. Busch, M. Koenig, J. Niegemann, "Discontinuous Galerkin methods in nanophotonics", Laser \& Photonics Reviews, 5(6), 2011, 773-809.

3. A. Taflove and S. C. Hagness, Computational Electrodynamics: The Finite-Difference Time-Domain Method, Artech House, 2005.

4. R. F. Remis, "On the stability of the finite-difference time-domain method", Journal of Computational Physics, 163(1), 2000, 249-261. 